Check for updates

Cite this: RSC Adv., 2018, 8, 37050

\title{
Bioinspired interconnected hydrogel capsules for enhanced catalysis $\uparrow$
}

\author{
Jiayao Chen, ${ }^{a}$ Minfeng Li, (D ${ }^{b}$ Wei Hong, ${ }^{a}$ Yuanjun Xia, ${ }^{c}$ Jingjing Lin*a \\ and Xudong Chen (DD *a
}

Received 22nd August 2018

Accepted 6th October 2018

Polysaccharide-based hydrogel capsules with interconnected inner membranes have been prepared. The obtained capsules have a multi-layer internal structure composed of many invaginations of interconnected hydrogel membranes as mimics of the cristae in mitochondria. These cristae-like internal membranes endow the capsules with high specific area, which makes the capsules ideal supporting materials for catalytic species such as metal nanoparticles and enzymes.

On the other hand, nature already provides an elegant example of highly efficient micro-reactor: mitochondria, a double membrane-bound organelle found in the cytoplasm of nearly all eukaryotic cells, has the main function to produce adenosine triphosphate (ATP). The curved inner membrane of mitochondria has many invaginations, known as the cristae, which greatly extended the surface areas of inner membrane and catalytic active species such as the ATP synthetase bound in the inner membrane..$^{12,13}$ Since heterogeneous reaction occurs on the surface, it is believed that the unique cristae structure with multi-layer inner membranes gives mitochondria high surface area which is responsible for the observed high productivity of mitochondria as a microreactor. $^{\text {14,15 }}$ Artificial multi-layered membrane hydrogels mimicking the hierarchical natural structures possess high specific area and controlled release property. ${ }^{16-19}$ The multilayered structure and tunable physical properties demonstrated a new generation of hydrogel materials. The hierarchical soft material with microstructures in different scales can be a prerequisite for the bio-related function and catalytic applications..$^{20,21}$ It could be envisioned that multilayered hydrogels could be an ideal substrate for MNPs catalysis.

Herein we report the design and fabricate of chitosan hydrogel capsules with interconnected inner membrane as a mimic of mitochondria's double-membrane structure by alkaline partial-stimulating method in aqueous solution. As a proof of concept, inner membranes of the resulting microreactors was decorated with catalytic active silver nanoparticles (Ag NPs) and the performance of the resulting microreactors was evaluated in reduction of 4-nitrophenol. We believed that the utilization of chitosan, a renewable and abundant biopolymer, to fabricate novel micro-reactors with high catalytic efficiency by an environmentally friendly process, shows the potential of sustainable applications.
${ }^{a}$ Key Laboratory for Polymeric Composite and Functional Materials of Ministry of
Education of China, Guangdong Engineering Technology Research Center for
High-performance Organic and Polymer Photoelectric Functional Films, School of
Chemistry, Sun Yat-sen University, Guangzhou 510275, China. E-mail: Linjj36@
mail.sysu.edu.cn; cescxd@mail.sysu.edu.cn
${ }^{b}$ College of Chemistry, Beijing Normal University, Beijing 100875, China
${ }^{c}$ Guangzhou General Hospital of Guangzhou Military Command, Guangzhou 510010,
China $\dagger$ Electronic supplementary information (ESI) available. See DOI: 10.1039/c8ra07037g 


\section{Experimental}

\subsection{Materials and methods}

2.1.1 Materials. Chitosan (CS, degree of deacetylation 85\%) was purchased from Sigma-Aldrich. Carboxymethylchitosan (CMCS, degree of carboxylation $80 \%$ ), silver nitrate $\left(\mathrm{AgNO}_{3}\right)$, sodium borohydride $\left(\mathrm{NaBH}_{4}\right)$, trisodium citrate dihydrate $\left(\mathrm{C}_{6}\right.$ $\mathrm{H}_{5} \mathrm{Na}_{3} \mathrm{O}_{7} \cdot 2 \mathrm{H}_{2} \mathrm{O}$ ), 4-nitrophenol (4-NP), methylene blue (MB), sodium hydroxide $(\mathrm{NaOH})$ and acetic acid $\left(\mathrm{CH}_{3} \mathrm{COOH}\right)$ were provided by local suppliers.

2.1.2 Preparation of interconnected hydrogel capsules. The hollow capsules were first prepared by adding a CS solution ( $2 \mathrm{wt} \%$ in $0.1 \mathrm{M}$, acetic acid) dropwise (with the nozzle diameter of 1.80 $\mathrm{mm}$ ) into CMCS aqueous solution (2 wt\%). The resulting hollow capsule with encapsulated CS solution was collected and subsequently rinsed with pure water. After that, the capsule was partially immersed in a $\mathrm{NaOH}$ solution $(2.5 \mathrm{wt} \%)$ for 1 minute, and transferred into pure water for 1 minute. Thus, by simply repeating the base treatment and sequential water soaking, multiple and interconnected CS gel membranes in the CS-CMCS hydrogel capsules could be generated. For comparison, solid hydrogel capsules were prepared by immersing the hollow capsules into ( $2.5 \mathrm{wt} \%) \mathrm{NaOH}$ solution for $10 \mathrm{~min}$.

2.1.3 Preparation of $\mathbf{A g}$ NPs loaded capsules. $\mathrm{Ag}$ NPs were in situ synthesized in chitosan hydrogel capsules with interconnected inner membranes or the solid hydrogel capsules. First, the capsules were immersed into $\mathrm{AgNO}_{3}$ solution $(50 \mathrm{ml}, 0.1 \mathrm{M}$ ) with magnetic stirring for $3 \mathrm{~h}$ at room temperature. During this process, $\mathrm{Ag}^{+}$was adsorbed evenly into the matrices of capsules through complexing with $-\mathrm{NH}_{2}$. Subsequently, the capsules with $\mathrm{Ag}^{+}$were collected and rinsed with distilled water for $20 \mathrm{~min}$. After that, the capsules were immersed into $50 \mathrm{ml} 1 \%(\mathrm{w} / \mathrm{v}) \mathrm{C}_{6}$ $\mathrm{H}_{5} \mathrm{Na}_{3} \mathrm{O}_{7} \cdot 2 \mathrm{H}_{2} \mathrm{O}$ aqueous solution with magnetic stirring for $2.5 \mathrm{~h}$ at $100{ }^{\circ} \mathrm{C}$. Due to the favorable mechanical and thermal properties, capsules were intact under vigorous stirring. Eventually, the dark Ag NPs loaded capsules were obtained, and rinsed with distilled water to remove excess reductive reagent.

2.1.4 Catalytic activity assays. The hydrogenation of 4-NP into 4-aminophenol (4-AP) by $\mathrm{NaBH}_{4}$ was chosen as the catalytic reaction. The reaction could be monitored by a UV-VIS spectrophotometer with a set time interval. First of all, 4-NP aqueous solution (25 ml, $0.1 \mathrm{mM}$ ) was mixed with $\mathrm{NaBH}_{4}$ aqueous solution (5 ml, $10 \mathrm{mM}$ ) in flask under stirring at room temperature, leading to a deep yellow solution. After the solution being mixed uniformly, $3 \mathrm{ml}$ of the solution was transferred to a centrifuge tube as a control group. Then, $18 \mathrm{Ag}$ NPs loaded capsules were added into $27 \mathrm{ml}$ of the solution with sustained shaking. For every three minutes, $3 \mathrm{ml}$ solution with 2 capsules were removed from the system for UV-VIS measurement.

Similarly, the reduction of MB dye was carried out as follows. MB dye aqueous solution ( $1.67 \mathrm{ml}, 0.1 \mathrm{mM})$ was mixed with $\mathrm{NaBH}_{4}$ aqueous solution $(1.33 \mathrm{ml}, 10 \mathrm{mM})$ in flask under stirring at room temperature, and the amount of the Ag NPs loaded hydrogel capsule was used the same as 2 capsules in $3 \mathrm{ml}$. The reduction of $\mathrm{MB}$ was monitored by the UV-VIS absorption spectroscopy.

\subsection{Characterization}

The solid-state ${ }^{13} \mathrm{C}$ nuclear magnetic resonance (NMR) spectra of capsules were recorded on a spectrometer operating at 400 $\mathrm{MHz}$ frequency $\left({ }^{13} \mathrm{C}-\mathrm{NMR}\right.$, Bruker Avance, Germany). The thickness of the capsule membrane was measured and calculated by field emission-scanning electron microscope (FESEM, Hitachi S-4800, Japan) with $10 \mathrm{kV}$ acceleration voltage. The appearance and structure of chitosan hydrogel capsules with interconnected inner membranes (dipping in water) were observed by a stereoscopic microscope (Micro optical instrument Co. Ltd. WSZ0745TB, China) under transmission light. The compression strength was measured on a universal tensile tester (SANT Test machine Co. Ltd. CMT6503, China) at a speed of $0.5 \mathrm{~mm} \mathrm{~min}^{-1}$. The specific surface area and the pore size distribution were measured by the BrunauerEmmett-Teller (BET) and Barrett-Joyner-Halenda (BJH) methods of nitrogen adsorption-desorption analyzer at $77 \mathrm{~K}$ (Quantachrome Autosorb-iQ2-MP, USA). To remove all the adsorbed species, the samples were degassed at $105{ }^{\circ} \mathrm{C}$ under vacuum. The morphology of Ag NPs loaded capsule was performed on FESEM and SEM coupled with energy dispersive $\mathrm{X}$ ray analysis (SEM-EDS, Hitachi S-4800, Japan). The high resolution transmission electron microscopy images were taken on a JEM-2010HR microscopy at $200 \mathrm{kV}$ (HRTEM, JEOL Co. Ltd JEM-2010HR, Japan). The compositions of capsules were measured with X-ray photo-electron spectroscopy (XPS, Thermofisher Scientific ESCALAB 250, USA) equipped with a monochromatic X-ray source ( $\mathrm{Al} \mathrm{K} \alpha, 1486.6 \mathrm{eV})$. The X-ray diffraction pattern of capsules were measured by X-ray diffractometer (XRD, Rigaku Co. Smart Lab, Japan). The catalytic activity of Ag NPs loaded capsules was measured via the reduction of 4-NP to 4-AP using UV/VIS spectrophotometer (UV-3600 Shimadzu Co., Japan). The thermal property of hydrogel capsules was evaluated by Thermo Gravimetry (TG, Netzsch TG20F1, Germany) with a heating rate of $10 \mathrm{~K} \mathrm{~min}^{-1}$ from 30 to $700{ }^{\circ} \mathrm{C}$. For all the tests, the samples were freezedried with lyophilizer (Alpha 1-4LSC CHRIST, Germany) at $-60{ }^{\circ} \mathrm{C}$ for $24 \mathrm{~h}$, except that the stereoscopic microscope observation, universal tensile and UV tests were measured in gel state.

\section{Results and discussion}

\subsection{Mechanism of interconnected hydrogel capsules}

The fabrication of chitosan hydrogel capsules with interconnected inner membranes involved two major steps: hollow capsules formation and sequential partial alkaline treatment of the capsules. Hollow capsules were first prepared by adding a CS (a positively charged polyelectrolyte) solution dropwise into CMCS (an amphoteric polyelectrolyte) aqueous solution. When the CS drops were added into the CMCS solution, a CSCMCS polyelectrolyte membrane was formed at the interface, isolating the encapsulated CS solution from further interacting with the surrounding CMCS solution. The thickness of CSCMCS membrane was measured by FE-SEM, which was about $10 \mu \mathrm{m}$ (Fig. S1, ESI $\dagger$ ). The composition of the CS-CMCS 


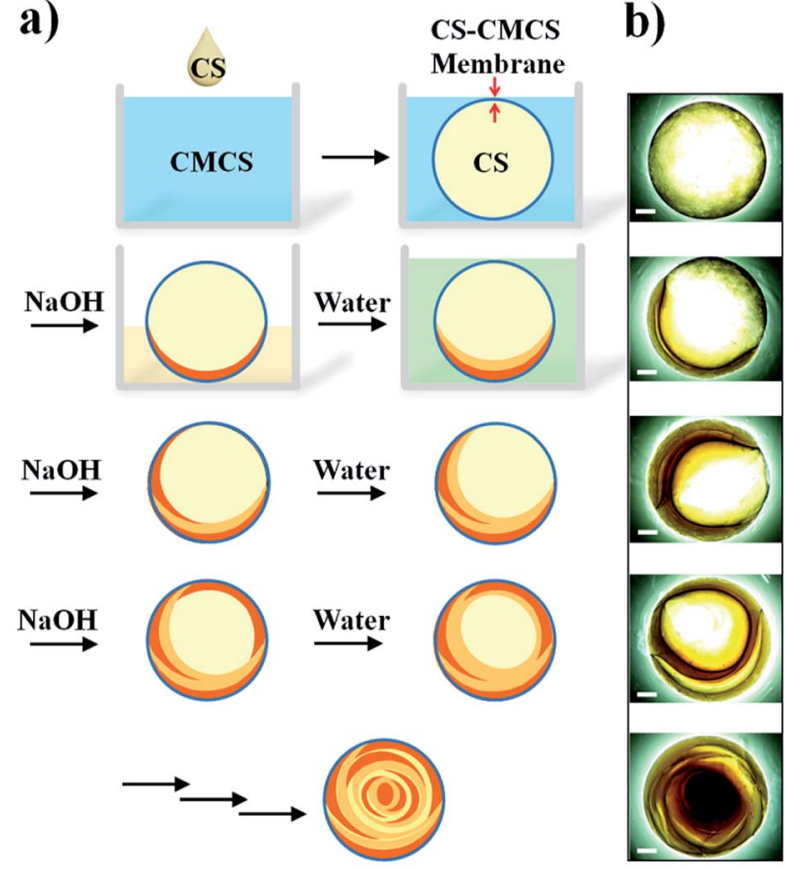

Scheme 1 (a) Schematic illustration of the fabrication of chitosan hydrogel capsules with interconnected inner membranes. (b) Stereoscopic microscope photographs of capsules at different stages. Scale bar: $1 \mathrm{~mm}$

membrane was further confirmed by ${ }^{13} \mathrm{C}$ NMR (Fig. S2, ESI $\dagger$ ) and XPS spectra (Fig. S3, ESI $\dagger$ ). After that, as illustrated in Scheme 1 , the capsule was partially immersed in a $\mathrm{NaOH}$ solution and transferred into pure water repeatedly. During this process, a thin layer of CS hydrogel membrane with a crescent shape could be observed inside the capsule. Thus, by simply repeating the base treatment and sequential water soaking, multiple and interconnected CS gel membranes in the CSCMCS hydrogel capsules could be generated (Scheme 1b). The optical images of the hollow capsules and hydrogel capsules with interconnected inner membranes were shown in Fig. S4 (ESI). $\dagger$

As expected for such a diffusion-reaction process, the thickness of newly formed inner gel membranes could be manipulated by varying the concentration of $\mathrm{NaOH}$ solution and the exposure time of the base treatment. As shown in Fig. 1a and $\mathrm{c}$, the thickness of the resulting inner gel membrane was positively related to the concentration of $\mathrm{NaOH}$ used for the base treatment. Similarly, it was found that thicker inner gel membranes could be obtained with prolonged treatment time (Fig. $1 \mathrm{~b}$ and $\mathrm{d}$ ). These observations implied that the base concentration gradient resulting from diffusion of $\mathrm{OH}^{-}$might play an important role in the formation of the inner gel membranes.

Fig. 2 indicated the mechanism of the capsule formation. The hollow capsule was partially exposed to basic solution, during which $\mathrm{OH}^{-}$passed through the permeable outer CSCMCS gel membrane and led to localized deprotonation of pendent $-\mathrm{NH}_{3}{ }^{+}$groups of $\mathrm{CS}$ in the exposed region, significantly
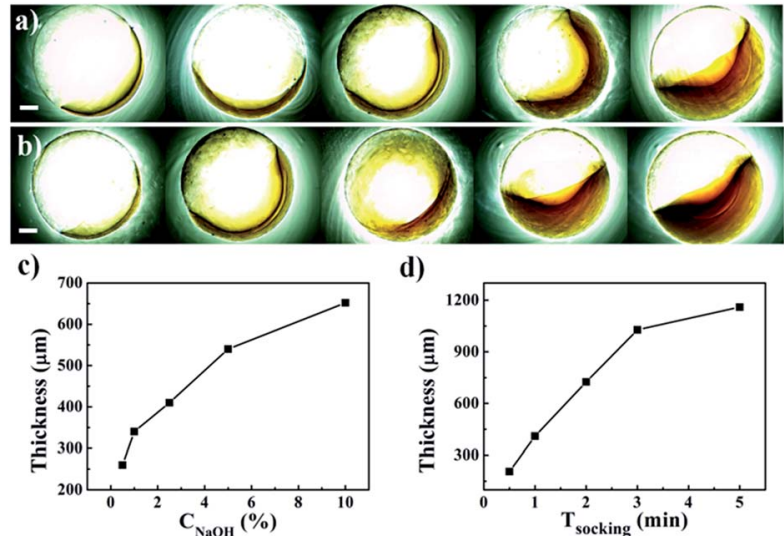

d)

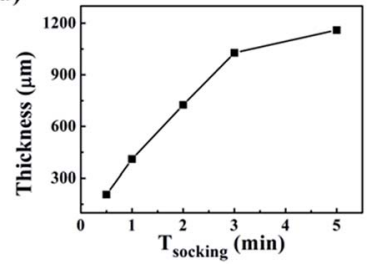

Fig. 1 The effects of the $\mathrm{NaOH}$ concentration and exposure time on the thickness of inner gel membranes: (a) photographs of CS-CMCS capsules treated by $\mathrm{NaOH}$ solution with concentration of $0.5,1,2.5,5$ and $10 \mathrm{wt} \%$ for $1 \mathrm{~min}$ (from left to right, respectively). (b) Photographs of CS-CMCS capsules treated by $\mathrm{NaOH}$ (2.5 wt\%) solution for $0.5,1,2$, 5 and 8 min, respectively. (c) Plots of the thickness of inner gel membranes versus concentration of $\mathrm{NaOH}$ solution and (d) exposure time. Scale bar: $1 \mathrm{~mm}$.

reducing the solubility of CS in this region. As a result, a crescent-shaped thin layer of CS gel membrane $\left(\mathrm{L}_{1}\right)$ appeared in (or "precipitate out of") the capsule (Fig. 2b). The subsequent water soaking of the capsule led to the diffusion of the excess $\mathrm{OH}^{-}$into the inner region and a crescent-shaped inner gel membrane $\left(L_{1}^{\prime}\right)$ with certain thickness was thus obtained (Fig. 2c). ${ }^{22}$ During this process, CS was neutralized incompletely due to the insufficient $\mathrm{OH}^{-}$. While in the second exposed process (hypothesis of overlap with $\mathrm{L}_{1} \& \mathrm{~L}_{1}^{\prime}$ ), the residual $-\mathrm{NH}_{3}{ }^{+}$ in $\mathrm{L}_{1}^{\prime}$ would be neutralized first (Fig. $2 \mathrm{~d}_{1}$ ) and a gap formed between the first two inner membranes, which could be attributed to the shrinkage of the CS volume due to the reduced electrostatic repulsion and recombination of hydrogen bond between CS molecules when the CS precipitated from the solution. After the formation of the $\mathrm{L}_{1}^{\prime}$ layer, $\mathrm{OH}^{-}$continuously diffused into inner capsule to form the third gel layer $\mathrm{L}_{2}$ (Fig. $2 \mathrm{~d}_{2}$ ). Through repeating solution treatment, additional
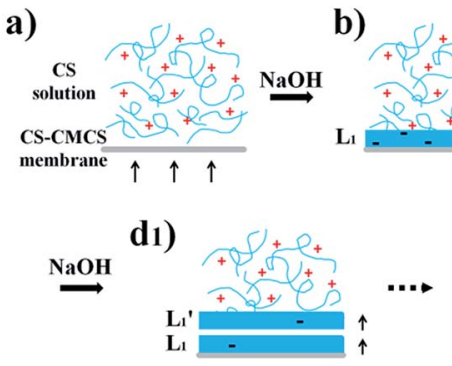

d2)

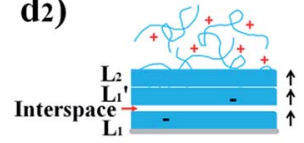

Fig. 2 Proposed mechanism of multi-layered gel membranes formation in the capsules: (a) hollow capsules partially exposed in $\mathrm{NaOH}$ solution; (b) and (c) inner gel layers formed in $\mathrm{NaOH}$ solution and water, respectively; $\left(d_{1}\right)$ the inter-membrane space stimulated by $\mathrm{NaOH}$ solution in second exposed process; $\left(d_{2}\right)$ the formation of the third gel layer fabricated. 
inner CS membranes could be readily formed. The interconnecting channels between the crescent-shaped inner membranes enabled small molecules to diffuse via the interconnected water-filled channels as a highway to quickly reach each crescent-shaped inner membrane inside the capsule and those molecules inside could also quickly find their way out of the capsule. The values of specific surface area and pore size were illustrated by $\mathrm{N}_{2}$ adsorption-desorption. Fig. S5† showed the $\mathrm{N}_{2}$ adsorption-desorption isotherms of the hydrogel capsules with interconnected inner membranes, which belonged to type II classification and $\mathrm{H} 3$ hysteresis loop. ${ }^{23}$ The values of specific surface area, total pore volume and pore size were calculated to be $36.742 \mathrm{~m}^{2} \mathrm{~g}^{-1}, 0.111 \mathrm{~cm}^{3} \mathrm{~g}^{-1}$ and $12.083 \mathrm{~nm}$, respectively, indicating the mesoporous feature of the hydrogel capsules. The value of surface area was higher than that of some former reported chitosan microspheres. ${ }^{24}$ The considerable physical properties of hydrogel capsules with interconnected inner membranes would greatly increase the matter transfer efficiency, resulting in enhanced productivity of the micro-reactors. In addition, the robust mechanical and thermal properties (Fig. S6†) of hydrogel capsules could be beneficial for the extreme catalytic environment.

\subsection{Morphology and structure of silver-loaded interconnected hydrogel capsules}

The interconnected hydrogel capsules with large surface areas and interconnecting channels have great potential to be highly effective carriers for catalytic species in the field of heterogeneous catalysis. As a proof of concept, Ag NPs were chosen to be immobilized on outer and inner membrane surfaces to fabricate a nanoparticles-gel composite as a micro-reaction system. The efficiency of this micro-reaction system would be evaluated by the reduction reaction of 4-NP to 4-AP in the next part. The Ag NPs loaded hydrogel capsules were prepared as follows: first, the capsules were treated with silver nitrate solution to immobilize $\mathrm{Ag}^{+}$ions to CS gel membranes though chelating interactions, and then Ag NPs were generated in situ in the presence of sodium borohydride as reducing reagent. ${ }^{25-27}$

The resulting nanoparticles-gel composite was characterized by FESEM and SEM-EDS to verify the presence of Ag NPs in membranes of capsules. FESEM photographs clearly showed that Ag NPs were successfully immobilized in the fibrous gel matrices with the diameters about $25 \mathrm{~nm}$ (Fig. 3a). Results of SEM-EDS analysis confirmed the presence of Ag NPs in the gel membranes (Fig. 3b).

The morphology and structure of Ag NPs in the membranes were also studied by TEM and HRTEM. TEM images showed that Ag NPs had a size of 20-25 nm (Fig. 4a), which were in consisted with the results obtained by FESEM. HRTEM and selected area electron diffraction (SAED) analysis showed clear lattice fringes which suggested that Ag NPs exhibited highly ordered crystalline-like structures (Fig. 4b). Result of XRD analysis of Ag NPs loaded gel membranes showed four distinct diffraction peaks at $38.24^{\circ}, 44.22^{\circ}, 64.68^{\circ}$ and $77.54^{\circ}$, which indexed the planes (111), (200), (220) and (311) of the cubic facecentered silver, respectively (Fig. 4d). While capsules membrane
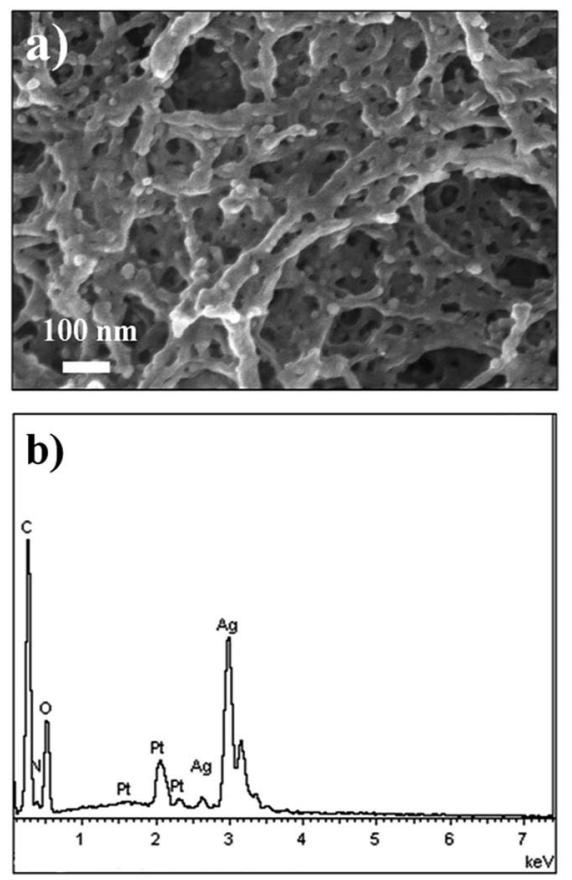

Fig. 3 (a) FESEM photograph and (b) SEM-EDS analysis of Ag NPs in the gel membranes of interconnected hydrogel capsules.

without Ag NPs showed only a broad peak at $22.42^{\circ}$ as a characteristic peak of amorphous chitosan (Fig. 4c).

As shown in the XPS spectra (Fig. 5a), the Ag 3d peak was observed in Ag NPs loading capsule. In comparison, the $\mathrm{Ag} 3 \mathrm{~d}$ peak was absent in those capsules without Ag NPs loading. The $\mathrm{Ag} 3 \mathrm{~d}$ peak in narrow scan XPS spectrum of Ag NPs loading capsule revealed two peaks (Fig. $5 \mathrm{~b}$ ): $\mathrm{Ag} 3 \mathrm{~d}_{5 / 2}$ and $\mathrm{Ag} 3 \mathrm{~d}_{3 / 2}$ with binding energy of 373.8 and $367.9 \mathrm{eV}$, respectively. Compared with the reported value of $\mathrm{Ag}^{0}$ peaks at 374.2 and $368.2 \mathrm{eV},{ }^{28}$ the observed $\mathrm{Ag}^{0}$ peaks slightly shifted to lower binding energy, which was believed as a result of the electron transfer from gel
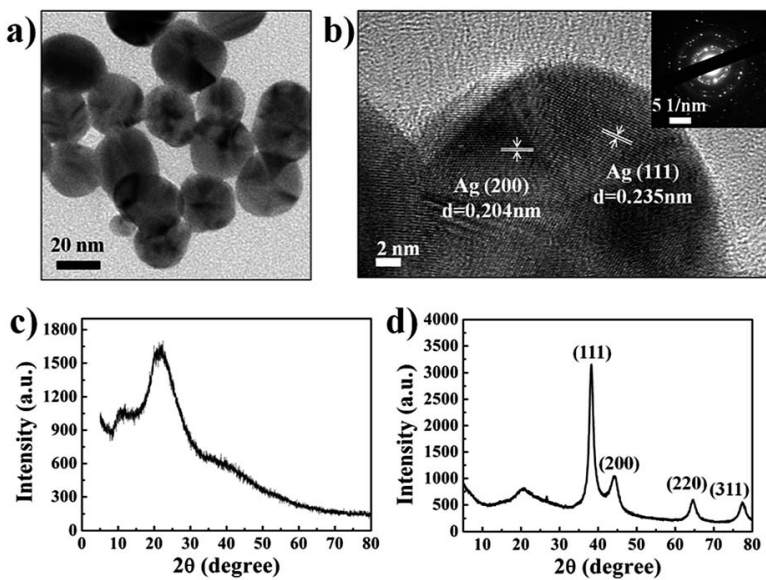

Fig. 4 Analysis of Ag NPs embedded in interconnected hydrogel capsules: (a) TEM image. (b) HRTEM image and (inset) SAED pattern. XRD spectra of the interconnected hydrogel capsules (c) without and (d) with loading Ag NPs. 

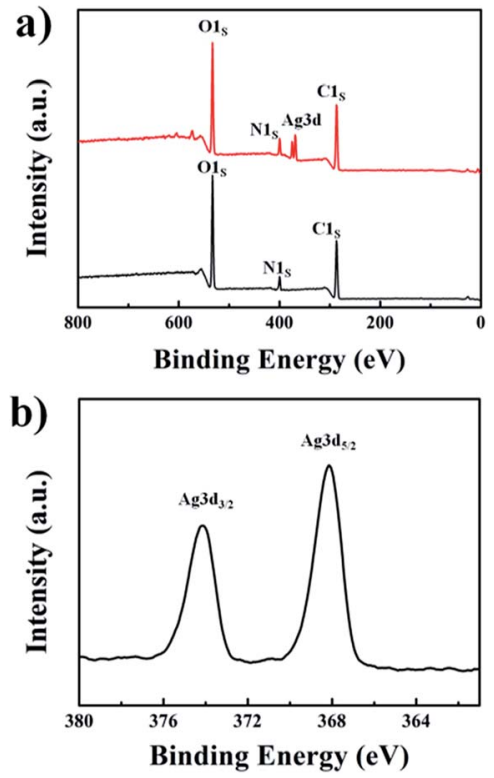

Fig. 5 (a) XPS spectra of interconnected hydrogel capsules (bottom, black line) and the capsules loading Ag NPs (top, red line). (b) XPS narrow scan spectrum of $\mathrm{Ag} 3 \mathrm{~d}$ peak.

matrix to the bound Ag NPs. ${ }^{24}$ XPS analysis also suggested that the content of $\mathrm{C}, \mathrm{N}, \mathrm{O}$ and $\mathrm{Ag}$ elements on the membrane surface were proximately $58.48,32.7,7.43$ and $1.39 \%$ respectively. The average Ag NPs mass content in capsules was estimated to be around $0.3105 \mathrm{mg}$ per interconnected hydrogel capsule ( $4.50 \mathrm{mg}$ per capsule in dry state in average) determined by ICP-AES.

\subsection{Catalytic activity}

As a model reaction, the catalytic reduction of 4-NP to 4-AP with sodium borohydride was used to investigate catalytic performance of Ag NPs loaded hydrogel capsules with interconnected inner membranes. The progress of the reaction was monitored by UV-VIS spectroscopy (Fig. 6a). The absorbance at $c a .400 \mathrm{~nm}$ was attributed to 4-NP ions in the presence of sodium borohydride. ${ }^{29}$ With the introduction of Ag NPs loaded hydrogel capsules into the reaction system, the absorbance at $400 \mathrm{~nm}$ gradually decreased with time and a new peak appeared at $290 \mathrm{~nm}$ which was attributed to the product 4-AP..$^{30,31}$ Sodium borohydride was present in large excess, so its concentration was considered as constant throughout the reaction. Thus reaction rates could be assumed to be independent of the concentration of sodium borohydride, which allowed that the pseudo-first-order kinetics with respect to 4-NP were employed to evaluate the reaction rate, ${ }^{32}$

$$
\ln \left(C / C_{0}\right)=-k t
$$

where $C$ and $C_{0}$ are the concentration of 4 -NP at reaction time $t$ and 0 , and $k$ is the apparent first-order rate constant. The $\ln (C /$ $C_{0}$ ) against the reaction time was plotted for the reduction of 4$\mathrm{NP}$, as depicted in Fig. 6b. A linear relation of the plots of $\ln (\mathrm{C} /$ $C_{0}$ ) versus reaction time were observed for the catalytic

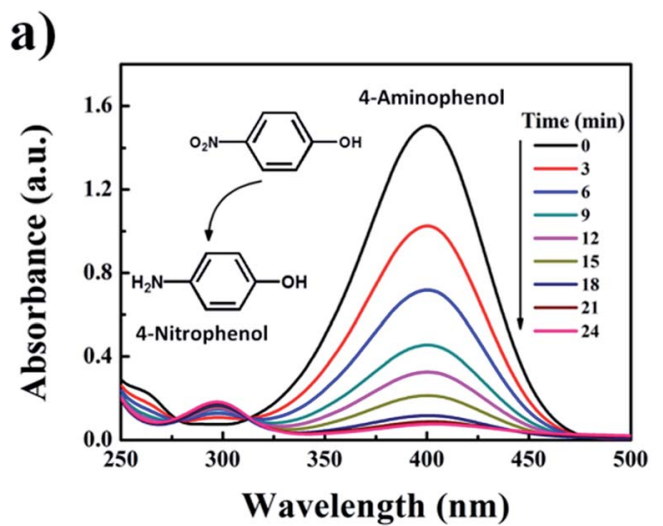

b)

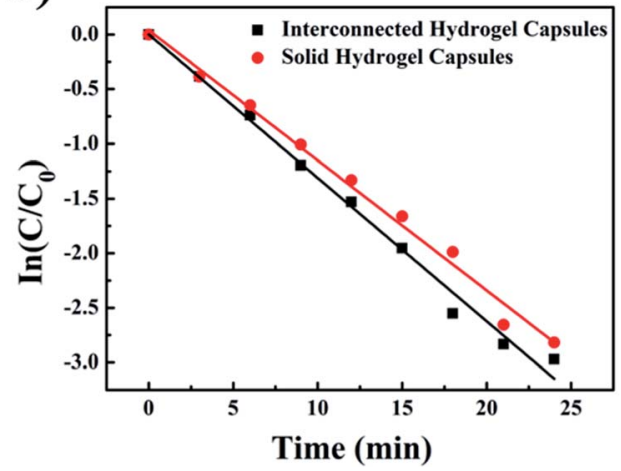

Fig. 6 (a) Representative UV/VIS spectra of 4-NP catalytic reduction with sodium borohydride in the presence of Ag NPs loaded interconnected hydrogel capsules as a function of time. (b) Representative plots of $\ln \left(C / C_{0}\right)$ against reaction time for the reduction of 4-NP by sodium borohydride in the presence of Ag NPs loaded interconnected hydrogel capsules and solid Ag NPs loaded hydrogel capsules at $400 \mathrm{~nm}$.

reduction of 4-NP with Ag NPs loaded hydrogel capsules, which suggested that the reaction followed pseudo-first order kinetics. The $k$ value was calculated based on the slope of the plotted line and the average value of $k$ was estimated to be $0.1312 \mathrm{~min}^{-1}$, which was comparable with some related works of catalytic hydrogel systems listed in Table 1. For comparison, the catalytic activity of solid Ag NPs loaded hydrogel capsules was determined to be $0.1189 \mathrm{~min}^{-1}$ (about $90 \%$ of the $k$ for multi-layered capsules), even though the solid hydrogel capsules were with higher Ag NPs loading (about $117 \%$ of the Ag amount for multilayered capsules) as shown in Table 1 . Due to the porosity of the hydrogel, the diffusion in the matrix was also essential as the solid hydrogel capsules exhibited a well catalytic activity, although the interconnected hydrogel capsules was more effective.

In order to evaluate the efficiency of the catalyst for a longterm use, the recycle experiment was performed with the $\mathrm{Ag}$ NPs loaded interconnected hydrogel capsules (see in Fig. S7†). The recyclability of the catalyst exhibited satisfactory activity $(>80 \%)$ after three cycles, and this macro-size hydrogels was beneficial for recycle and reuse. In addition, the significant reduction of MB was also shown in Fig. S8, $\dagger$ supporting the generic catalytic activity of the Ag NPs loaded hydrogel capsules. 
Table 1 Comparison of kinetic rate constants $(k)$ value of different catalytic hydrogel systems for $\mathrm{NaBH}_{4}$ reducing 4-NP in the recent literatures and our work

\begin{tabular}{|c|c|c|c|c|c|}
\hline Catalyst & $c 4-\mathrm{NP}(\mathrm{M})$ & MNPs loading $\left(\mathrm{mg} \mathrm{L}^{-1}\right)$ & Amount of catalyst & $k\left(\min ^{-1}\right)$ & Ref. \\
\hline Ag NPs-embedded semi-IPN hydrogel & $2 \times 10^{-4}$ & 622 & $10 \mathrm{mg}$ in $2.7 \mathrm{ml}$ & 0.374 & 33 \\
\hline Alginate-based biohydrogels (Ag@AMH) & $9.9 \times 10^{-5}$ & - & $\begin{array}{l}\text { Four beads in } \\
2.525 \mathrm{ml}\end{array}$ & 0.27 & 34 \\
\hline Poly(NIPAAm-co-AMPS) hydrogel-Ag NPs & $2.16 \times 10^{-3}$ & - & - & $0.0642\left(26^{\circ} \mathrm{C}\right)$ & 35 \\
\hline DNA hydrogel-Au NPs & $2 \times 10^{-4}$ & - & $100 \mathrm{mg}$ in $1 \mathrm{ml}$ & 0.09 & 27 \\
\hline P(AMPS) hydrogel-Ni NPs & $7.19 \times 10^{-4}$ & 118.4 & $50 \mathrm{mg}$ in $50 \mathrm{ml}$ & $0.0563\left(30^{\circ} \mathrm{C}\right)$ & 26 \\
\hline
\end{tabular}

The result demonstrated that the Ag NPs loaded hydrogel capsules with interconnected inner membranes exhibited decent catalytic performance in the reduction of 4-NP comparing with reported catalysts in macro-hydrogel system.

\section{Conclusions}

In conclusion, it was demonstrated that hydrogel capsules with cristae-like internal membranes could be prepared from polysaccharides with an iterative base-water treatment protocol. As a proof of concept, Ag NPs were immobilized in the capsules to fabricate a nanoparticles-gel composite as a micro-reaction system for catalytic reduction of 4-nitrophenol to 4-aminophenol. Ag NPs loaded interconnected hydrogel capsules showed excellent catalytic performance in the reduction of 4nitrophenol. Giving their mitochondria-like unique features such as large reactive surface areas and good diffusion feature, these hydrogel capsules should have great potential to be highly effective carriers for catalytic species in the development of environmental friendly heterogeneous catalytic reaction systems.

\section{Conflicts of interest}

There are no conflicts to declare.

\section{Acknowledgements}

This work was supported by the National Natural Science Foundation of China (Grant No. 51233008), the Natural Science Foundation of Guangdong Province of China (Grant No. 2014A030311035) and Guangdong YangFan Innovative \& Entrepreneurial Research Team Program.

\section{Notes and references}

1 D. Astruc, F. Lu and J. R. Aranzaes, Angew. Chem., Int. Ed., 2005, 44, 7852.

2 L. Liu and A. Corma, Chem. Rev., 2018, 118, 4981.

3 R. Narayanan and M. A. El-Sayed, J. Am. Chem. Soc., 2003, 125, 8340.

4 M. Hermanek, R. Zboril, I. Medrik, J. Pechousek and C. Gregor, J. Am. Chem. Soc., 2007, 129, 10929.
5 A. Shahzad, W. S. Kim and T. Yu, RSC Adv., 2015, 5, 28652.

6 B. Léger, S. Menuel, A. Ponchel, F. Hapiot and E. Monflier, Adv. Synth. Catal., 2012, 354, 1269.

7 H. Hu, J. H. Xin, H. Hu, X. Wang, D. Miao and Y. Liu, J. Mater. Chem. A, 2015, 3, 11157.

8 Y. Lu, P. Spyra, Y. Mei, M. Ballauff and A. Pich, Macromol. Chem. Phys., 2007, 208, 254.

9 D. D. Díaz, D. Kühbeck and R. J. Koopmans, Chem. Soc. Rev., 2011, 40, 427.

10 T. R. Chetia, M. S. Ansari and M. Qureshi, Phys. Chem. Chem. Phys., 2016, 18, 5344.

11 N. Sahiner, Prog. Polym. Sci., 2013, 38, 1329.

12 T. G. Frey and C. A. Mannella, Trends Biochem. Sci., 2000, 25, 319.

13 S. Cogliati, C. Frezza, M. E. Soriano, T. Varanita, R. Quintana-Cabrera, M. Corrado, S. Cipolat, V. Costa, A. Casarin, L. C. Gomes, E. Perales-Clemente, L. Salviati, P. Fernandez-Silva, J. A. Enriquez and L. Scorrano, Cell, 2013, 155, 160.

14 C. A. Mannella, Biochim. Biophys. Acta, 2006, 1762, 140.

15 G. A. Perkins, J. Tjong, J. M. Brown, P. H. Poquiz, R. T. Scott, D. R. Kolson, M. H. Ellisman and G. A. Spirou, J. Neurosci., 2010, 30, 1015.

16 B. C. Zarket and S. R. Raghavan, Nat. Commun., 2017, 8, 193.

17 N. Lin, A. Gèze, D. Wouessidjewe, J. Huang and A. Dufresne, ACS Appl. Mater. Interfaces, 2016, 8, 6880.

18 Y. Xiong, K. Yan, W. E. Bentley, H. Deng, Y. Du, G. F. Payne and X.-W. Shi, ACS Appl. Mater. Interfaces, 2014, 6, 2948.

19 M. He, Y. Zhao, J. Duan, Z. Wang, Y. Chen and L. Zhang, ACS Appl. Mater. Interfaces, 2014, 6, 1872.

20 J. Nie, W. Lu, J. Ma, L. Yang, Z. Wang, A. Qin and Q. Hu, Sci. Rep., 2015, 5, 7635.

21 S. Cho, Y. Li, M. Seo and E. Kumacheva, Angew. Chem., Int. Ed., 2016, 55, 14014.

22 S. Ladet, L. David and A. Domard, Nature, 2008, 452, 76.

23 M. Kruk and M. Jaroniec, Chem. Mater., 2001, 13, 3169.

24 B. Duan, F. Liu, M. He and L. Zhang, Green Chem., 2014, 16, 2835.

25 S. Butun and N. Sahiner, Polymer, 2011, 52, 4834.

26 N. Sahiner, H. Ozay, O. Ozay and N. Aktas, Appl. Catal., A, 2010, 385, 201.

27 A. Zinchenko, Y. Miwa, L. I. Lopatina, V. G. Sergeyev and S. Murata, ACS Appl. Mater. Interfaces, 2014, 6, 3226. 
28 D. Lin, H. Wu, R. Zhang and W. Pan, Chem. Mater., 2009, 21, 3479.

29 S. Jana, S. K. Ghosh, S. Nath, S. Pande, S. Praharaj, S. Panigrahi, S. Basu, T. Endo and T. Pal, Appl. Catal., A, 2006, 313, 41.

30 A. A. Antipov, G. B. Sukhorukov, Y. A. Fedutik, J. Hartmann, M. Giersig and H. Möhwald, Langmuir, 2002, 18, 6687.

31 S. K. Ghosh, M. Mandal, S. Kundu, S. Nath and T. Pal, Appl. Catal., A, 2004, 268, 61.
32 S. K. Das, M. M. R. Khan, A. K. Guha and N. Naskar, Green Chem., 2013, 15, 2548.

33 M. V. Patwadkar, C. S. Gopinathbc and M. V. Badiger, RSC $A d v .$, 2015, 5, 7567.

34 L. Ai, H. Yue and J. Jiang, J. Mater. Chem., 2012, 22, 23447.

35 M. Wang, J. Wang, Y. Wang, C. Liu, J. Liu, Z. Qiu, Y. Xu, S. F. Lincoln and X. Guo, Colloid Polym. Sci., 2016, 294, 1087. 